\title{
Estratégia da saúde da família: perfil dos médicos e enfermeiros, Londrina, Paraná
}

\author{
Family health teams: profile of the physicians and nurses in \\ Londrina, Paraná, Brazil
}

\author{
Rosângela Aparecida Pimenta Ferrari ${ }^{1}$; Zuleika Thomson²; Regina Melchior ${ }^{2}$
}

\section{Resumo}

Trata-se de um estudo descritivo quantitativo, com o objetivo de descrever o perfil dos médicos e enfermeiros das equipes da Saúde da Família, no município de Londrina, Paraná. A coleta de dados foi realizada no período de agosto a setembro de 2003 e os dados foram analisados através do programa EpiInfo. O município contava com 188 profissionais ( 94 médicos e 94 enfermeiros). Desse total, 89 enfermeiros $(94,7 \%)$ e 82 médicos $(87,2 \%)$ participaram da pesquisa. Mais da metade dos médicos era do sexo masculino, com média de idade de 42,2 anos. Entre os enfermeiros, predominou o sexo feminino, com média de idade de 33,9 anos. Grande parte dos médicos atuava há mais de nove anos na área da Saúde Coletiva, enquanto a atuação de mais da metade dos enfermeiros, nessa área, era de menos de nove anos. Quase a totalidade dos enfermeiros e pouco mais de $50 \%$ dos médicos tinham mais de dois anos de trabalho na ESF. Mais de $74 \%$ dos médicos e $93,2 \%$ dos enfermeiros haviam feito especialização, referindo terem-na feito na área da Saúde Coletiva, respectivamente $45 \%$ e $70 \%$. Diante destes resultados verifica-se que os médicos e os enfermeiros das equipes da Saúde da Família do município de Londrina possuíam expressiva qualificação profissional.

Palavras-chave: Programa Saúde da Família. Atenção básica de saúde. Recursos humanos em saúde.

\begin{abstract}
This study is a descriptive quantitative investigation to describe the profile of the members of the family health teams in Londrina, Paraná. Data were collected in August and September, 2003. In order to analyze quantitative data, the Epi-Info program was used. The city of Londrina reckoned on 188 health professionals (94 physicians and 94 nurses), out of which 89 nurses $(94,7 \%)$ and 82 physicians $(87,2 \%)$ took part in this research. Over fifty percent of the physicians were male, the average age being 42,2 years old. Among the nurses, the female prevailed, average age being 33,9 years old. Most of the physicians had already worked for more than 9 years in the collective health area, while most of the nurses had worked in this area for less than 9 years. Almost all the nurses and about 50\% of the physicians had worked for more than two years in ESF (Family Health Teams). Concerning formal education, more than $74 \%$ of the physicians and $93,2 \%$ of the nurses answered that they had completed graduate courses: $45 \%$ of the physicians and $70 \%$ of the nurses, in Collective Health. In face of these results, it was verified that the physicians and the nurses of the family health teams in Londrina had significant professional qualification.
\end{abstract}

Key words: Family Health Program. Basic health care. Human resource in health.

\footnotetext{
1 Professora, Mestre em Saúde Coletiva do Departamento de Enfermagem (Saúde da Criança e do Adolescente) da Universidade Estadual de Londrina - PR, Brasil. Endereço Profissional: Av. Robert Koch, n. 60, Vila Operária, CCS - Departamento de Enfermagem, Fone/Fax: 3371-2309, $1^{\circ}$ andar, CEP: 86038-440, Londrina-PR; E-mail: ropimentaferrari@uel.br.

2 Professoras, Doutoras em Saúde Pública do Departamento de Saúde Coletiva da Universidade Estadual de Londrina - PR, Brasil.
}

Semina: Ciências Biológicas e da Saúde, Londrina, v. 26, n. 2, p. 101 -108, jul./dez. 2005 


\section{Introdução}

A estratégia da Saúde da Família - SF foi implantada em todo o país como importante mudança de política pública de saúde para reordenar o modelo assistencial, priorizando-se ações de promoção, proteção e recuperação da saúde dos indivíduos e da família de forma integral e contínua.

Para a concretização da implantação dessa estratégia, fez-se necessário formar profissionais para a abordagem do processo de saúde/doença com enfoque em Saúde da Família, que compreende dois focos: o da Saúde da Família, relativo ao estado de saúde dos indivíduos que a compõem, e o do funcionamento, que é uma descrição avaliativa das funções e estruturas da família e constitui, portanto, um quadro no qual o foco da avaliação e da assistência está na saúde como um todo (ANGELO; BOUSSO, 2001).

Assim sendo, a responsabilidade dos profissionais estará voltada para a assistência integral e contínua de todos os membros das famílias da população adscrita à unidade de saúde, em cada uma das fases do seu ciclo da vida, não se restringindo, dessarte, a uma atuação em face dos problemas de saúde biologicamente presentes, mas como compromisso que envolve as ações dos indivíduos enquanto saudáveis.

Nesta proposta, a SF elege como ponto central o estabelecimento de vínculos e a criação de laços de compromisso e de responsabilidade entre os profissionais e a população e, assim, propõe trabalhar na perspectiva da vigilância à saúde, com responsabilidade integral para com a população que reside na área de abrangência de suas unidades de saúde (BRASIL, 1998).

Assim, a Saúde da Família é colocada como uma estratégia desenvolvida para promover mudanças no atual modelo de assistência à saúde do país, possibilitando, que efetivamente, sejam colocados em prática os princípios que norteiam o SUS, como: integralidade da assistência, universalidade, eqüidade, participação e controle social, intersetorialidade, resolutividade, saúde como direito e humanização do atendimento.

Para tanto, lidar com estas inovações e adaptálas às diferenças locais e regionais é necessário que os profissionais das equipes da Saúde da Família estejam capacitados e esta pesquisa teve como objetivo descrever o perfil dos médicos e dos enfermeiros que atuam nesta estratégia no município de Londrina.

\section{Metodologia}

Trata-se de uma investigação descritiva transversal quantitativa. Este estudo foi desenvolvido nas UBS de Londrina, localizada no Norte do Paraná, região Sul do Brasil.

Londrina possui 447.065 habitantes, dos quais 96,9\% residem na área urbana (LONDRINA, 2003).

No município de Londrina, em 1994, iniciou-se o Programa Médico de Família, com quatro equipes implantadas nos distritos e na zona rural. Em dezembro de 1995, assumiu a gestão semiplena pela NOB-93 (Norma Operacional Básica) e em janeiro de 1998, a gestão plena do Sistema Municipal de Saúde, com a edição da NOB-96. Em maio de 2001, a nova gestão municipal ampliou as equipes, antes Programa Médico de Família, de 4 para 81 equipes de Saúde da Família. No início de 2002, houve ampliação de mais 12 equipes. Em abril de 2003 foi aprovada mais uma equipe, totalizando 94. Esta ampliação fez com que a população da área rural tivesse $100 \%$ de cobertura e $70 \%$ a da área urbana. $\mathrm{Na}$ área urbana, as periferias e zonas de maior risco como os bolsões de pobreza e áreas de assentamento, etc., foram priorizadas tendo cobertura de 100\% (LONDRINA, 2003a).

As 94 equipes de Saúde da Família estavam assim distribuídas nas 50 UBS: 9 na zona rural e 85 na zona urbana. Cada equipe era formada por nove profissionais: um médico, um enfermeiro, dois auxiliares de enfermagem e cinco agentes comunitários de saúde. 
A população do estudo foi constituída por médicos e enfermeiros das UBS que atuavam nas equipes da Saúde da Família, no período de agosto a outubro do ano de 2003. A coleta de dados foi realizada por meio de um formulário com perguntas fechadas de múltipla escolha. Os formulários foram entregues para cada profissional para serem preenchidos no local de trabalho, sendo recolhidos posteriormente.

A análise das respostas dos formulários foi realizada pelo programa Epi-Info 6.04d (DEAN et al., 1995).

A pesquisa foi analisada e aprovada pelo Comitê de Ética em Pesquisa da Universidade Estadual de Londrina - UEL, parecer CEP 176/02, em 11 de dezembro de 2001.

\section{Resultados e Discussão}

Os resultados a seguir apresentados dizem respeito aos 171 formulários preenchidos pelos médicos e enfermeiros das equipes da Saúde da Família do município de Londrina, no período de agosto a outubro do ano de 2003.

Foram coletados dados nas 50 Unidades Básicas de Saúde - UBS que possuíam 94 equipes da Saúde da Família, totalizando 188 profissionais (94 médicos e 94 enfermeiros). Do total desses profissionais, $87,2 \%$ (82) médicos e 94,7\% (89) enfermeiros participaram da pesquisa.

Com relação ao perfil dos profissionais, a Tabela 1 demonstra que foi predominante o sexo masculino entre os médicos e o sexo feminino entre os enfermeiros.

Tabela 1 - Distribuição dos médicos e dos enfermeiros das equipes da Saúde da Família, segundo o sexo, Londrina, 2003.

\begin{tabular}{cccrrrr}
\hline \multirow{2}{*}{ Sexo } & \multicolumn{2}{c}{ Médicos } & \multicolumn{2}{c}{ Enfermeiros } & \multicolumn{2}{c}{ Total } \\
\cline { 2 - 7 } & $\mathrm{N}^{\mathrm{o}}$ & \multicolumn{1}{c}{$\%$} & \multicolumn{1}{c}{$\mathrm{N}^{\mathrm{o}}$} & \multicolumn{1}{c}{$\%$} & \multicolumn{1}{c}{$\mathrm{N}^{0}$} & $\%$ \\
\hline Masculino & 49 & 59,8 & 5 & 5,6 & 54 & 31,6 \\
Feminino & 33 & 40,2 & 84 & 94,4 & 117 & 68,4 \\
\hline Total & 82 & 100,0 & 89 & 100,0 & 171 & 100,0 \\
\hline
\end{tabular}

Quanto à idade, 43,9\% dos médicos e 73\% dos enfermeiros tinham menos de 40 anos de idade, uma média de 42,2 anos e 33,9 anos, respectivamente.

Os resultados deste estudo quanto ao sexo e idade dos enfermeiros foram semelhantes aos dados da pesquisa de Machado (2000) acerca do perfil dos médicos e enfermeiros da SF, no Brasil. Nessa pesquisa, realizada em 1998, verificou-se que a grande maioria dos enfermeiros era constituída por mulheres com menos de 40 anos de idade. Outra pesquisa, realizada por Teixeira (2002) em toda região de Ribeirão Preto/SP, mostrou que a totalidade dos enfermeiros da SF era do sexo feminino.

Com relação aos médicos, os dados da pesquisa de Machado (2000) também foram semelhantes ao deste estudo, apontando que mais da metade dos médicos que trabalhava na SF, no Brasil, era do sexo masculino com mais de 40 anos de idade.

Os resultados desta pesquisa reafirmam ainda a enfermagem como uma profissão predominantemente feminina, mas parece que o número de homens na área está aumentando, com o passar dos anos.

Com relação à área médica, ainda há predomínio do sexo masculino, mas, neste estudo, $40 \%$ dos profissionais eram do sexo feminino.

Machado (1998) pesquisou, em 1996, o perfil dos médicos no Brasil e concluiu que a medicina da atualidade experimenta grandes mudanças na prática profissional. Neste cenário, salientam-se três fatos: o rejuvenescimento, a urbanização e a feminilização. Primeiro, os médicos que tinham menos de 45 anos de idade representavam quase um terço de toda classe médica. Segundo, a concentração cada vez mais acentuada nos grandes centros urbanos. Terceiro, a rápida e irreversível feminilização da área médica.

Quanto à atuação profissional, os resultados deste estudo demonstraram que grande parte dos médicos $(62,2 \%)$ e cerca de $46 \%$ dos enfermeiros tinham mais de nove anos de tempo de profissão, com média de 16 anos e 9,2 anos, respectivamente. 
Quanto ao tempo de atuação em UBS, aproximadamente $60 \%$ dos médicos (média de 9,1 anos) e apenas $37 \%$ dos enfermeiros (média de 5,8 anos) tinham mais de cinco anos. A grande maioria dos enfermeiros $(80,9 \%)$ e mais da metade dos médicos $(56,1 \%)$ tinham mais de dois anos de trabalho na Saúde da Família, o que dá uma média de 2,4 e 2,0 anos, respectivamente.

Pode-se observar que o tempo de profissão e o tempo de atuação em UBS foi maior entre os médicos do que entre os enfermeiros, enquanto o maior tempo de atuação na equipe da Saúde da Família foi maior entre os enfermeiros do entre os médicos.

Girardi e Carvalho (2003) referem que com a implantação da SF no país ocorreram novas perspectivas de trabalho e modalidades de contratação. Com isso, alterou-se o perfil do mercado de trabalho na área, com um acréscimo de mais de $20 \%$ de empregos no setor público.

No caso do emprego ofertado aos médicos, o estudo de Machado (1998) demonstrou que 70\% dos médicos tinham vínculo com o setor público no âmbito municipal, estadual ou federal, e poucos eram os que dependiam de emprego em instituições privadas.

Provavelmente, o maior vínculo no setor público pelos médicos foi devido aos incentivos e benefícios oferecidos na época da implantação do Programa da Saúde da Família, como altos salários e promessas de capacitação, que, sem dúvida, levaram muitos a abandonarem suas áreas de atuação para trabalharem no programa (JANAUDIS, 2003).

Pode-se verificar que um grande percentual de enfermeiros tinha mais de dois anos de trabalho no programa, enquanto na pesquisa de Teixeira (2002) apenas $34 \%$ dos enfermeiros tinham mais de dois anos de trabalho na SF, e cerca de 56\% trabalhava há menos de um ano.

O contrato de serviço dos profissionais das equipes da SF do município era pelo regime celetista ou estatutário. Mais da metade dos enfermeiros (57,3\%) trabalhava sob o regime celetista e $50 \%$ dos médicos tinham contrato celetista, e a outra metade era integrada por estatutários.

Entre as modalidades de contratação sugeridas pelo Ministério da Saúde para as equipes da Saúde da Família consta também o contrato celetista, que deve revestir-se das garantias e dos direitos jurídicotrabalhistas de cada profissional contratado no serviço, nesta modalidade.

A contratação pelo regime celetista por instituição filantrópica alcançou quase $70 \%$ do total de contratos de médicos e enfermeiros dos municípios da Região Sudeste, e superou $60 \%$ nos municípios da região Sul, sendo de $70 \%$ a porcentagem referente ao Brasil (GIRARDI; CARVALHO, 2003).

Estes autores referiram também que quase $2 / 3$ dos empregos gerados na estratégia não se revestiram dos graus de formalização e institucionalidade das relações de trabalho típicas do setor público e do mercado de trabalho privado formal, pois desconsideraram não apenas direitos trabalhistas consagrados, como também ameaçaram a própria continuidade da prestação de serviço com qualidade.

Machado (2000) apresentou dados semelhantes aos de Girardi e Carvalho (2003) em que mais de $40 \%$ dos médicos e enfermeiros do país foram incorporados por meio de contratos temporários. Segundo essa mesma pesquisa, cerca de $60 \%$ destes profissionais não possuíam garantias jurídicas de direitos trabalhistas.

Cabe salientar que, ao contrário das pesquisas de Machado (2000), Girardi e Carvalho (2003), o município de Londrina tem assegurado os direitos trabalhistas dos profissionais que são contratados pelo regime celetista (LONDRINA, 2003a).

Quanto à situação de estar contratado pelo regime celetista, Machado (2000) também mostrou que quase metade dos profissionais referiu que essa situação constituía-se um fator estressante e de instabilidade.

Quanto à instabilidade no emprego por contratos que não asseguram os direitos trabalhistas, Andrade 
(1998) refere que esta situação pode flexibilizar as relações de trabalho na estratégia, comprometendo sua continuidade e, principalmente, a relação com a comunidade no que diz respeito, por exemplo, a sua credibilidade e da Secretaria de Saúde que adota esse sistema.

A situação de instabilidade faz com que os profissionais procurem outras oportunidades de emprego o que, conseqüentemente, compromete e adia os compromissos e metas firmadas durante as gestões, diminuindo a qualidade da prestação de serviços à população desta região.

Para mudar este quadro, Souza et al. (2002, p. 27) sugerem a necessidade de:

[...] avançar na direção da sustentabilidade das transformações do modelo de atenção. É preciso que estes elementos sejam potencializados com processos mais efetivos de gestão, medidas que visem a fixação das equipes em seus municípios, ampliação de recursos com responsabilidade tripartite, políticas de recursos humanos que promovam valorização social do trabalho dos profissionais que trabalham na área da saúde pública, com destaque para os que compõem as equipes da Saúde da Família e, maior ousadia nas instituições de ensino para inovar na formação de seus profissionais.

Com relação ao fato de os enfermeiros serem coordenadores da equipe da ESF, aproximadamente $37 \%$ dos enfermeiros referiram não ser coordenadores da equipe em que trabalhavam. Tal fato reflete-se na própria organização do serviço, mostrando que, possivelmente, não tenham sido enfatizadas as atividades desenvolvidas na coordenação das equipes.

Quanto às funções e atribuições dos profissionais da estratégia, o Ministério da Saúde determina aos enfermeiros a responsabilidade de coordenar, gerenciar, treinar e administrar a unidade de saúde (BRASIL, 1998).

Os dados desta pesquisa foram semelhantes aos da pesquisa realizada por Girardi e Carvalho (2003), na qual cerca de $30 \%$ dos enfermeiros contratados para trabalhar no Programa da Saúde da Família referiram também não coordenar a equipe.

Cabe salientar, também, que cerca de $2,4 \%$ dos médicos afirmaram ser coordenadores da equipe. Provavelmente esses profissionais também não receberam esclarecimentos quanto às atribuições dos outros profissionais da equipe, por exemplo, quanto a ser a coordenação função do enfermeiro.

Com relação à formação dos profissionais das equipes da Saúde da Família, verifica-se na Tabela 2 que a grande maioria dos médicos e enfermeiros afirmou ter feito pós-graduação.

Dos profissionais que referiram ter feito pósgraduação, 20,7\% dos médicos e 52,8\% dos enfermeiros tinham feito uma especialização e, 56,1\% médicos e $41,6 \%$ enfermeiros tinham feito mais de uma especialização.

Tabela 2 - Distribuição dos médicos e dos enfermeiros das equipes da Saúde da Família, segundo a realização de pós-graduação, Londrina, 2003.

\begin{tabular}{lrrrr}
\hline \multirow{2}{*}{ Pós-graduação } & \multicolumn{2}{c}{ Médicos } & \multicolumn{2}{c}{ Enfermeiros } \\
\cline { 2 - 5 } & \multicolumn{1}{c}{$\mathrm{N}^{\mathrm{o}}$} & $\%$ & $\mathrm{~N}^{\mathrm{o}}$ & $\%$ \\
\hline Lato sensu & 61 & 74,4 & 83 & 93,2 \\
Nenhuma & 19 & 23,2 & 5 & 5,6 \\
Stricto sensu & 2 & 2,4 & 1 & 1,2 \\
\hline \multicolumn{1}{c}{ Total } & 82 & 100,0 & 89 & 100,0 \\
\hline
\end{tabular}

O estudo de Machado (2000) demonstrou que pouco mais de $39 \%$ dos médicos e $35 \%$ dos enfermeiros que trabalhavam na SF, no Brasil, referiram ter alguma especialização lato sensu.

Cabe salientar que, comparando-se o estudo de Machado (2000) com este estudo, vê-se que, neste último, quase o dobro dos médicos e dos enfermeiros referiu ter feito pós-graduação lato sensu.

Quanto à pós-graduação stricto sensu entre os profissionais das equipes, os dados da pesquisa da mesma autora foram semelhantes aos deste estudo, diferindo em pouco mais de $2 \%$. 
Com relação à área da pós-graduação cursada pelos médicos, a Tabela 3 mostra que a maior porcentagem das respostas foi primeiramente para a área de Clínica Médica, seguida da área de Saúde Coletiva e Materno-Infantil.

Dos 29 (46\%) médicos que responderam ter feito pós-graduação na área da Saúde Coletiva, 18 (62,1\%) tinham-na feito em Saúde da Família e 11 (37,9\%) em Saúde Coletiva. Quanto à área Materno-Infantil, dos 20 (31,7\%) médicos 08 (40\%) eram pediatras e $12(60 \%)$, ginecologistas e obstetras.

Tabela 3 - Distribuição dos médicos das equipes da Saúde da Família, segundo a área da pós-graduação lato sensu e stricto sensu, Londrina, 2003.

\begin{tabular}{lcc}
\hline \multicolumn{1}{c}{ Área da pós-graduação } & \multicolumn{2}{c}{ Médicos $=63^{* *}$} \\
lato sensu e stricto sensu & \multicolumn{2}{c}{$\%$} \\
\cline { 2 - 3 } & $\mathrm{N}^{\mathrm{o}}$ & $\%$ \\
\hline Clínica Médica & 48 & 76,2 \\
Saúde Coletiva & 29 & 46,0 \\
Mateno-Infantil & 20 & 31,7 \\
Clínica Cirúrgica & 8 & 12,7 \\
Área não especificada & 7 & 11,1 \\
\hline
\end{tabular}

* Permite múltiplas respostas

** \% calculada com base no $\mathbf{n}$

A Tabela 4 demonstra que quase $70 \%$ dos enfermeiros referiram ter feito a pós-graduação na área da Saúde Coletiva.

Destes, 23 (41,1\%) referiram ter feito o curso de especialização em Saúde Coletiva, 21 (37,5\%) em Saúde da Família e 12 (21,4\%) em Gerência de Unidades Básicas de Saúde - GERUS.
Tabela 4 - Distribuição dos enfermeiros das equipes da Saúde da Família, segundo a área da pós-graduação lato sensu e stricto sensu, Londrina, 2003.

\begin{tabular}{lcr}
\hline Área da pós-graduação & \multicolumn{2}{c}{ Enfermeiros $=84 * *$} \\
\cline { 2 - 3 } lato sensu e stricto sensu* & $\mathrm{N}^{\mathrm{o}}$ & $\%$ \\
\hline Saúde Coletiva & 56 & 66,7 \\
\hline Educação & 40 & 47,6 \\
\hline Hospitalar & 19 & 22,6 \\
\hline Área não especificada & 1 & 1,2 \\
\hline
\end{tabular}

* Permite múltiplas respostas

** \% calculada com base no $\mathbf{n}$

Verifica-se que foi expressivo o número de médicos e enfermeiros deste estudo que fizeram pós-graduação na área da Saúde Coletiva.

Quanto aos enfermeiros, os dados deste estudo foram semelhantes aos da pesquisa de Thumé (2001), no Rio Grande do Sul, que observou que a maioria (60\%) dos enfermeiros da rede básica de saúde tinha feito curso de pós-graduação na área da Saúde Coletiva.

Por outro lado, o estudo de Teixeira (2002) mostrou que apenas $17 \%$ dos enfermeiros tinham especialização. Já os enfermeiros que não a possuíam informaram sentirem-se desatualizados e necessitarem de atualização em nível de pósgraduação em Saúde da Família, pois na graduação não tiveram nenhum embasamento sobre essa estratégia.

O investimento na pós-graduação é necessário para a qualificação dos profissionais, mas também deve haver maiores investimentos na formação acadêmica dos futuros profissionais.

Quanto a este fato Souza et al. (2002, p. 27) referiram:

[...] se os profissionais de saúde saírem de suas instituições formadoras interessados e preparados para atuar na atenção básica com a mesma disposição que saem hoje para atuar num centro de alta tecnologia e especialização, poderão estabelecer o fortalecimento do processo de construção de um sistema de saúde mais justo e equânime. 
Ainda para essas autoras, os mesmos profissionais recém-egressos também deverão encontrar serviços de atenção básica valorizados, reconhecidos socialmente e com capacidade de assegurar-lhes a manutenção da sua escolha. Desse modo, eles sentirse-ão dignos de prestar serviços de atenção básica às pessoas, famílias e comunidades. Se as instituições formadoras atenderem às necessidades dos futuros profissionais com base nos princípios do SUS, poderão também contribuir para atuação dos médicos e enfermeiros, facilitando-lhes a prestação de serviços com qualidade a toda população.

\section{Considerações Finais}

Cabe ressaltar que este estudo teve por objetivo fazer uma descrição do perfil dos médicos e enfermeiros das equipes da Saúde da Família do município de Londrina. Certamente, a pesquisa poderá contribuir para o aprofundamento da temática, com a realização de novas pesquisas utilizando outras metodologias.

Quanto aos resultados obtidos, verificou-se que, do total dos profissionais, foi predominante o sexo masculino entre os médicos, e o feminino entre os enfermeiros. Grande parte dos profissionais tinha menos de 40 anos de idade, com mais de nove anos de atuação na área da atenção básica de saúde, enquanto o tempo de atuação no Programa da Saúde da Família girava em torno de dois anos sendo os médicos, em sua maioria, estatutários, enquanto os enfermeiros eram celestitas. Frente aos resultados, sugere-se a necessidade do reconhecimento e valorização da qualificação dos profissionais que trabalham no Programa da Saúde da Família em Londrina, pois, caso se compare Londrina com outros municípios, os profissionais deste estudo apresentaram significativa qualificação pelo tempo de trabalho dedicado à atenção básica de saúde, na Saúde da Família e por serem pós-graduados na área da Saúde Pública, demonstrando o potencial humano já existente para atender a população londrinense.

\section{Agradecimentos}

Aos profissionais da Diretoria de Atenção à Saúde da Autarquia Municipal de Saúde de Londrina: Rosália Mestre Okabayashi, por agilizar os trâmites da pesquisa nas UBS, Marilda Kohatsu e Sonia Regina Nery, por disponibilizarem informações necessárias à pesquisa; às alunas do curso de graduação em Enfermagem da Universidade Estadual de Londrina: Bruna Camila F. da Silva, Fernanda Donádio Pitta e Giseli Plath pelo empenho e colaboração para recolher os formulários em cada UBS; aos enfermeiros coordenadores das UBS, por facilitarem o acesso aos médicos e enfermeiros das equipes da Saúde da Família; e de forma especial, aos médicos e enfermeiros que se dispuseram a responder aos formulários. Sem a participação de cada um a pesquisa não teria sido viabilizada.

\section{Referências}

ANGELO, M.; BOUSSO, R. S. Fundamentos da assistência a família em saúde. In: BRASIL. Instituto para o Desenvolvimento da Saúde. Universidade São Paulo. Ministério da Saúde. Manual de Enfermagem. Brasília: Ministério da Saúde, 2001.

ANDRADE, F.M.O. O Programa de saúde da família no Ceará: uma análise de sua estrutura e funcionamento. Fortaleza: Expressão, 1998.

BRASIL. Secretaria da Saúde de Assistência a Saúde. Coordenação de Saúde da Comunidade. Saúde da Família: uma estratégia para a reorientação do modelo assistencial. Brasília, 1998.

DEAN, A.G.; DEAN, J. A, COULOMBIER, D.; BRENDEL, K.A.; SMITH, D.C.; BURTON, A.H.; DICKER, R. C.; SULLIVAN, K.; FAGAN, R. F; ARNER, T. G.Epi Info: version $6.04 \mathrm{~d}$ a Word processing, database and statistics program for epidemiology on microcomputers. Atlanta: Centers for Disease Control and Prevention, 1995.

GIRARDI, S.N.; CARVALHO, C.L. Contratação e qualidade do emprego no programa de saúde da família no Brasil. In: FALCÃO, A. et al. (Orgs). Observatório de recursos humanos em saúde no Brasil: estudos e análises. Rio de Janeiro: FIOCRUZ, 2003. p. 157-190.

JANAUDIS, M. A. Medicina de Família ou Programa de Saúde da Família?. IN: RONCOLETA, A. F. T. et al. Princípios da Medicina de Família. São Paulo: SOBRAMFA - Soc. Bras. de Med. de Família, 2003. cap. 5. 
LONDRINA. Secretaria Municipal de Planejamento. Sintese do perfil do município de Londrina 2003:baseado nos dados de 2002. Londrina, 2003. Disponível em:<http:/ /www.londrina.pr.gov.br/perfil2003.htm>. Acesso em: 10 fev. 2004.

LONDRINA. Prefeitura Municipal. Projeto municipal de expansão da saúde da familia: PROESF. Londrina, 2003a.

MACHADO, M.H. A profissão médica no contexto de mudanças. In: GOULART, F.A. de A.; CARVALHO, G. de C. M. (Orgs.). Os médicos e a saúde no Brasil. Brasília: Conselho Federal de Medicina, 1998. p.181-200.

MACHADO, M.H. Perfil dos médicos e enfermeiros do programa saúde da família no Brasil: relatório final. Brasília, DF: Ministério da Saúde, 2000. v.1 - Brasil e grandes regiões.
SOUZA, H.M. et al. A implantação de uma nova mentalidade. Revista Brasileira Saúde Família, Brasília, ano 2, n.6, p. 20-27, dez. 2002.

TEIXEIRA, R.A. O trabalho da enfermeira na saúde da família: potência de (re)construção do modelo assistencial e (re)criação do trabalho da enfermagem? 2002.. Dissertação (Mestrado em Enfermagem) - Escola de Enfermagem de Ribeirão Preto, Universidade de São Paulo, Ribeirão Preto.

THUMÉ, E. Enfermagem em atenção básica na saúde coletiva: práticas da especialidade na região sul do Rio Grande do Sul. Div. Saúde para Debate, Rio de Janeiro, n.23, p. 64-73, dez. 2001. 